\title{
INFLUENCE OF MUSCLE MASS AREA AND VISCERAL OBESITY ON 30-DAY MORTALITY AFTER COLORECTAL SURGERY WITH PRIMARY ANASTOMOSIS
}

\author{
Paulina Moctezuma-Velázquez ${ }^{1}$, Omar Vergara-Fernández ${ }^{1}$, Noel Salgado-Nesme ${ }^{1}$, \\ Jorge L. Aguilar-Frasco ${ }^{1}$, Juan C. Sainz-Hernández ${ }^{1}$, and Carlos Moctezuma-Velázquez ${ }^{2 *}$
}

Departments of ${ }^{1}$ Surgery and ${ }^{2}$ Gastroenterology, Instituto Nacional de Ciencias Médicas y Nutrición Salvador Zubirán, Mexico City, Mexico

\begin{abstract}
Background: Muscle mass and visceral fat may be assessed at the level of the third lumbar vertebra (L3) in computed tomography (CT). Both variables have been related with adverse surgical outcomes. Objective: The objective of the study was to study the association of skeletal muscle index (SMI) and visceral fat area (VFA) with 30-day mortality in colorectal surgery. Methods: This is a retrospective cohort study conducted at a tertiary referral hospital in Mexico City. Patients who underwent colorectal surgery with primary anastomosis from January 2007 to December 2018 were included in the study. Their preoperative CT scans were analyzed with the NIH Image] software at the level of the third lumbar vertebra to determine their SMI (L3-SMI) and the VFA. Logistic regression analysis (adjusted by surgery anatomical location) was used to determine the association between these variables and surgical 30-day mortality. Results: A total of 548 patients were included; 30-day mortality was 4.18\% (23 patients). On univariable analysis, L3-SMI, low SMI, anastomosis leak, pre-operative albumin, estimated blood loss, age, steroid use, Charlson comorbidity index score $>2$, and type of surgery were associated with 30 -day mortality. On multivariable analysis, low SMI remained an independent risk factor with an odds ratio of $4.74,95 \%$ confidence interval 1.22-18.36 ( $p=0.02$ ). Conclusion: Low SMI was found to be an independent risk factor for 30-day mortality in patients submitted to colorectal surgery with a primary anastomosis, whether for benign or malignant diagnosis. VFA was not associated with 30-day mortality. (REV INVEST CLIN. 2021;73(6):379-87)
\end{abstract}

Key words: Sarcopenia. Skeletal muscle. Visceral fat. Colorectal surgery.

\section{BACKGROUND}

Body composition analysis by computed tomography (CT) has been studied in different medical outcomes.
Adipose and muscle tissue distribution are strongly correlated to the patients' metabolic and performance status, respectively; both features could influence surgical outcomes. The visceral fat area (VFA) and
*Corresponding author:

Carlos Moctezuma-Velázquez

E-mail: mocmocte@hotmail.com
Received for publication: 22-02-2021

Approved for publication: 12-05-2021

DOI: $10.24875 / R I C .21000108$

0034-8376 / (c) 2021 Revista de Investigación Clínica. Published by Permanyer. This is an open access article under the CC BY-NC-ND license (http://creativecommons.org/licenses/by-nc-nd/4.0/). 
skeletal muscle area (SMA) may be assessed by the quantification of each tissue in a transversal CT image at the third lumbar vertebra (L3) with excellent inter and intraobserver agreement. Both variables have been identified as prognostic factors for oncologic and surgical outcomes among colorectal cancer surgery patients. The use of image analysis to determine fat and muscle distribution is an advantage because CT is widely available among this population ${ }^{1,2}$.

Visceral obesity has been associated with longer operative times and hospital stays, as well as with increased morbidity ${ }^{3,4}$. Low skeletal muscle mass is also known to have a negative effect on post-operative outcomes ${ }^{5}$, mainly among patients with cancer, increasing their morbidity and mortality. Most data regarding SMA and adipose tissue analysis focus on the oncological population, but there is a lack of information regarding patients with benign entities. The importance of identifying the implications of SMA and VFA in surgical outcomes relies on the implementation of interventions that target SMA and VFA to improve these outcomes.

Sarcopenia is defined by the Sarcopenia Working Group as a syndrome characterized by the loss of skeletal muscle mass and strength ${ }^{6}$. Since we only analyzed muscle area and not function, we decided not to use the term sarcopenia; instead, the term SMA or skeletal muscle index (SMI) will be used accordingly. SMI is obtained by dividing the patients' SMA by their height.

The objective was to study the association of VFA and SMI with 30-day mortality in patients undergoing any colorectal surgery including primary anastomosis. A secondary aim was to analyze the association between these variables and anastomosis leak ( $A L)$.

\section{METHODS}

\section{Study Population}

This study is a retrospective analysis of all patients who underwent colorectal surgery with primary anastomosis from January 2007 to December 2018 at a tertiary referral hospital in Mexico City. We excluded
54 patients who had incomplete medical charts since the variables needed for the study could not be obtained from the electronic or physical files or because no pre-operative CT was available. Finally, only 548 patients were included in the study. All surgeries were performed by the two chief surgeons of our colorectal surgery department.

\section{Data Collection}

For each patient, the following variables were collected: (1) patient characteristics, including age, sex, preoperative albumin, diagnosis (cancer or benign), steroid use, current smoker, comorbidities calculated with Charlson comorbidity index (CCI) score, weight, height, body mass index (BMI), VFA, and SMA; (2) operative details, including the type of surgery (right hemicolectomy, left hemicolectomy, surgeries with rectal anastomosis, and others) blood loss, type of case (urgent or elective surgery), number of anastomoses, type of anastomosis (manual or stapled), approach (open or laparoscopic), conversion, protective stoma, and operative time; and (3) post-operative outcomes: AL, length of stay, 30-day readmission, and 30-day mortality. This study was approved by our institution's Research Ethics Committee with the reference number GAS 318419201.

\section{Body Composition Analysis}

Pre-operative CTs were assessed with $\mathrm{NIH}$ Image] software, version $1.48^{7}$. VFA and SMA were assessed at the level of L3. VFA was computed with a Hounsfield unit (HU) threshold between -30 and $-190 \mathrm{HU}$, whereas SMA with a threshold between -29 and +150 HU. The L3-SMI was calculated by dividing SMA/ height $\left(\mathrm{cm}^{2}\right)$. Visceral obesity was defined as a VFA $>100 \mathrm{~cm}^{2}$, and low SMI was diagnosed if patients had an $\mathrm{SMl}<52.4 \mathrm{~cm}^{2} / \mathrm{m}^{2}$ and $<38.5 \mathrm{~cm}^{2} / \mathrm{m}^{2}$ in men and women, respectively ${ }^{8}$. Agreement between raters to classify patients as having or not having low SMI was assessed with Cohen's kappa. We also computed the intraclass correlation coefficient of the estimated SMA ${ }^{9}$.

\section{Statistical Analysis}

Statistical analysis was performed using Stata 14 (StataCorp, Texas, USA). Continuous variables were 
Table 1 . Frequency of low SMI and visceral obesity by BMI $\left(\mathrm{cm} / \mathrm{m}^{2}\right)$ category

\begin{tabular}{lcccc}
\hline Characteristics & $\begin{array}{c}\text { BMI <18.5 } \\
(\mathrm{n}=31)\end{array}$ & $\begin{array}{c}\text { BMI 18.5-24.9 } \\
(\mathrm{n}=246)\end{array}$ & $\begin{array}{c}\text { BMI 25-29.9 } \\
(\mathrm{n}=205)\end{array}$ & $\begin{array}{c}\text { BMI>30 } \\
(\mathrm{n}=66)\end{array}$ \\
\hline Low SMla, $\mathrm{n}(\%)$ & $20(64)$ & $153(62)$ & $99(48)$ & $14(21)$ \\
Visceral obesity, $\mathrm{n}(\%)$ & $2(6)$ & $88(36)$ & $147(72)$ & $58(88)$ \\
\hline
\end{tabular}

aDefined as an SMI at the level of $\mathrm{L} 3$ of $<52.4 \mathrm{~cm}^{2} / \mathrm{m}^{2}$ in men and $<38.5 \mathrm{~cm}^{2} / \mathrm{m}^{2}$ in women.

bDefined as visceral fat area $>100 \mathrm{~cm}^{2}$.

BMI: body mass index; SMI: skeletal muscle index.

presented as means \pm standard deviations and categorical variables as frequencies $(n)$ and percentages. Variables were compared using the Chi-squared test or Fisher's exact test, and Student's t-test as appropriate. To study the association between body composition variables and the outcomes of interest (i.e., 30-day mortality and $\mathrm{AL}$ ), we used logistic regression. Those variables with $p<0.05$ were included in a multivariable analysis. We used the Box-Tidwell model to test the linearity of continuous variables and goodness-of-fit was assessed by the Hosmer-Lemeshow test.

\section{RESULTS}

\section{General Characteristics of Patients}

The study population consisted of 548 patients with a mean age of $57.4+17.4$ years; $262(47.7 \%)$ were male. The prevalence of low SMI was $52 \%$ (286 patients), and it was higher among men ( $60 \%$ vs. $45 \%$, $p<0.001$ ). The prevalence of visceral obesity was $53.7 \%$ (295 patients) and was also more common in men $(69 \%$ vs. $40 \%, p<0.001)$.

According to the $\mathrm{BMI}$, among the overweight and obese patients, low SMI was found in $99(48 \%)$ and $14(21 \%)$, respectively; visceral obesity was found in $88(36 \%)$ patients with normal BMI (Table 1 ). The prevalence of visceral obesity in conjunction with low SMI (i.e., low SMI and high VFA) was $28 \%$; when obesity was defined by the BMI (i.e., >30) instead of VFA, the prevalence was $2.5 \%$. The mean BMI was $25.2+$ $5.1 \mathrm{~kg} / \mathrm{m}^{2}$.

The most common surgery was right hemicolectomy in 174 patients (32.5\%). Two hundred and eighteen
(40\%) cases were laparoscopic, and the conversion rate was $35 \%$. Two hundred and seventy-six patients (50\%) had colorectal cancer. The rest of the variables is described in Table 2.

Patients with low SMI were older $(60.4+18.2$ vs. $54.2+16.0$ years; $\mathrm{p}<0.001)$, had a lower BMI $(23.9$ +3.8 vs. $\left.26.8+5.8 \mathrm{~kg} / \mathrm{m}^{2} ; \mathrm{p}<0.001\right)$, and had a higher 30 -day mortality ( $6.6 \%$ vs. $1.5 \%$; $p=0.003$ ) than patients with a normal SMI. There were no differences in length of stay, 30-day readmission, or frequency of AL. Compared to non-obese patients, those with visceral obesity were more commonly men $(62 \%$ vs. $32 \% ; p<0.001)$ and were older $(60.2+$ 15.3 vs. $54.1+19.1$ years; $p<0.001$ ). Table 3 compares patients according to their body composition. The 30-day mortality was similar in patients with and without visceral obesity in conjunction with low SMI ( $6.5 \%$ vs. $3.3 \% ; p=0.09$ ). Cohen's kappa coefficient for the assessment of low SMI was $0.76(p<0.001)$, and the intraclass correlation coefficient for SMA was $0.96(p<0.001)$.

\section{Features Associated with Mortality and $\mathrm{AL}$}

Thirty-day mortality was $4 \%$. On univariable analysis (Table 4), factors associated with mortality were low SMI (odds ratio [OR] 4.59, 95\% confidence interval [CI] 1.54-13.67; $p=0.006$ ), the L3-SMI (OR 0.92, $95 \% \mathrm{Cl} 0.87-0.97 ; \mathrm{p}=0.003), \mathrm{AL}(\mathrm{OR} 8.62,95 \% \mathrm{Cl}$ 3.57-20.82; $<<0.001$ ), pre-operative albumin (OR $0.34,95 \% \mathrm{Cl}, 0.20-0.56 ; \mathrm{p}<0.001)$, estimated blood loss (OR 1.00, 95\% Cl 1.00-1.001; $\mathrm{p}=0.02$ ), age (OR 1.06, $95 \% \mathrm{Cl} 1.03-1.09 ; \mathrm{p}<0.001$ ), $\mathrm{CCl}$ score $>2$ (OR 10.30, 95\% Cl 3.75-28.26; $p<0.001)$, steroid use (OR 5.33, 95\% Cl 1.83-15.48; $\mathrm{p}=0.002$ ), and other type of surgery (i.e., total colectomy, 
Table 2. General population characteristics $(n=548)$

\begin{tabular}{|c|c|}
\hline Patient characteristics & Total \\
\hline Male, n (\%) & $262(48)$ \\
\hline Age (years), mean + SD & $57.4+17.4$ \\
\hline Low SMla, n (\%) & $286(52)$ \\
\hline Visceral obesity ${ }^{\mathrm{b}}, \mathrm{n}(\%)$ & $295(54)$ \\
\hline BMI $\left(\mathrm{kg} / \mathrm{m}^{2}\right)$, mean + SD & $25.3+5.1$ \\
\hline Cancer diagnosis, n (\%) & $276(50)$ \\
\hline $\begin{array}{l}\text { Pre-operative albumin }(\mathrm{g} / \mathrm{dL}) \text {, } \\
\text { mean }+\mathrm{SD}\end{array}$ & $3.8+0.7$ \\
\hline Current smoker, n (\%) & $230(42)$ \\
\hline Steroid use, n (\%) & $31(6)$ \\
\hline \multicolumn{2}{|l|}{ Operative details } \\
\hline \multicolumn{2}{|l|}{ Type of surgery, n (\%) } \\
\hline Right hemicolectomy & $174(32)$ \\
\hline Sigmoidectomy & $128(23)$ \\
\hline Stoma closure & $75(14)$ \\
\hline Total colectomy & $25(4)$ \\
\hline Left hemicolectomy & $37(7)$ \\
\hline Low anterior resection & $42(8)$ \\
\hline Proctocolectomy + J pouch & $26(5)$ \\
\hline Others ${ }^{c}$ & $116(21)$ \\
\hline $\begin{array}{l}\text { Estimated blood loss }(\mathrm{mL}) \\
\text { mean }+\mathrm{SD}\end{array}$ & $374.5+371.5$ \\
\hline Emergency surgery, $\mathrm{n}(\%)$ & $92(17)$ \\
\hline $\begin{array}{l}\text { Surgical time (minutes), } \\
\text { mean + SD }\end{array}$ & $247.0+94.7$ \\
\hline Manual anastomosis, n (\%) & $59(11)$ \\
\hline Laparoscopic surgery, n (\%) & $218(40)$ \\
\hline Conversion, $\mathrm{n}(\%)$ & $71(35)$ \\
\hline Loop ileostomy, n (\%) & $157(29)$ \\
\hline \multicolumn{2}{|l|}{ Post-operative outcomes } \\
\hline 30-day readmission, $\mathrm{n}(\%)$ & $38(7)$ \\
\hline 30-day mortality, n (\%) & $23(4)$ \\
\hline $\mathrm{AL}, \mathrm{n}(\%)$ & $53(9.7)$ \\
\hline \multicolumn{2}{|l|}{ Clavien-Dindo, n (\%) } \\
\hline I & $76(14.10)$ \\
\hline$\|$ & $67(12.43)$ \\
\hline IIIA & $21(3.90)$ \\
\hline IIIB & $33(6.12)$ \\
\hline IVA & $42(7.79)$ \\
\hline IVB & $25(4.64)$ \\
\hline $\mathrm{V}$ & $23(4)$ \\
\hline
\end{tabular}

BMI: body mass index; SMI: skeletal muscle index;

SD: standard deviation; AL: anastomosis leak.

aDefined as an SMI at the level of $\mathrm{L} 3$ of $<52.4 \mathrm{~cm}^{2} / \mathrm{m}^{2}$ in men and $<38.5 \mathrm{~cm}^{2} / \mathrm{m}^{2}$ in women.

befined as visceral fat area $>100 \mathrm{~cm}^{2}$.

'Stoma closure and segmental resection. proctocolectomy, sigmoidectomy, and low anterior resection) (OR $2.78,95 \% \mathrm{Cl} 0.95-8.07 ; \mathrm{p}=0.06$ ). On multivariable analysis (Table 4), low SMI remained an independent risk factor for 30-day mortality as well as $\mathrm{AL}$, age, steroid use, $\mathrm{CCl}>2$, pre-operative serum albumin levels, estimated blood loss, and other types of surgery. The same variables were associated with 30-day mortality when including L3-SMI instead of low $\mathrm{SMI}$ in the multivariable analysis.

AL developed in 53 (9.7\%) patients; blood loss, $\mathrm{CCl}>2$, and other types of surgery were variables associated with $\mathrm{AL}$ in univariable and multivariable analyses (Table 5).

A sub-analysis of geriatric and oncological patients can be found in Supplementary Tables 1 and 2 .

\section{DISCUSSION}

Body composition assessment through radiological evaluation has become an important pre-operative tool to identify patients with increased risk of poorer surgical outcomes. In this study, we investigated the association between SMA, assessed with the SMI, and VFA with 30-day mortality among colorectal surgery patients.

In this work, we found low SMI to be independently associated with 30-day mortality but not with AL. On the other hand, neither visceral obesity nor visceral obesity in conjunction with low SMI was associated with any of these outcomes. To the best of our knowledge, this is the first study comparing the influence of both visceral obesity and SMI on post-operative outcomes of patients undergoing colorectal surgery for oncologic and benign disorders in a Mexican population.

The prevalence of low SMI in our population was $52 \%$, and $54 \%$ of patients had visceral obesity. Despite the high prevalence of low SMI in oncological patients, no difference in low SMI prevalence was found between oncological and non-oncological patients in our study. This underlines the importance of assessing muscle mass and function in all surgical patients, and not only in elderly or oncological patients. 
Table 3. General characteristics by body composition

\begin{tabular}{|c|c|c|c|c|c|c|}
\hline Variable & $\begin{array}{l}\text { Normal SMI } \\
(n=262)\end{array}$ & $\begin{array}{l}\text { Low SMIa } \\
(n=286)\end{array}$ & $\mathrm{p}$-value & $\begin{array}{l}\text { No visceral } \\
\text { obesity } \\
(n=253)\end{array}$ & $\begin{array}{l}\text { Visceral obesity } \\
\quad(n=295)\end{array}$ & p-value \\
\hline \multicolumn{7}{|l|}{ Pre-operative characteristics } \\
\hline Male, n (\%) & $105(40)$ & $157(55)$ & $<0.001$ & $80(32)$ & $182(62)$ & $<0.001$ \\
\hline Age (years), mean + SD & $54.2+16.0$ & $60.4+18.2$ & $<0.001$ & $54.1+19.1$ & $60.2+15.3$ & $<0.001$ \\
\hline $\begin{array}{l}\text { Charlson comorbidity } \\
\text { index }>2, \mathrm{n}(\%)\end{array}$ & $119(45)$ & $156(54)$ & 0.03 & $120(47)$ & $155(52)$ & 0.2 \\
\hline Cancer diagnosis, $\mathrm{n}(\%)$ & $134(51)$ & $142(50)$ & 0.7 & $132(52)$ & $144(49)$ & 0.2 \\
\hline BMI $\left(\mathrm{kg} / \mathrm{m}^{2}\right)$, mean + SD & $26.8+5.8$ & $23.9+3.8$ & $<0.001$ & $22.8+3.8$ & $27.4+5.1$ & $<0.001$ \\
\hline Albumin $(\mathrm{g} / \mathrm{dL})$, mean + SD & $3.8+0.8$ & $3.8+0.7$ & 0.4 & $3.7+0.8$ & $3.9+0.7$ & 0.07 \\
\hline \multicolumn{7}{|l|}{ Surgical characteristics } \\
\hline $\begin{array}{l}\text { Surgical time (min), } \\
\text { mean + SD }\end{array}$ & $252.9+91.2$ & $241.6+97.5$ & 0.2 & $242.9+94.1$ & $250.5+95.2$ & 0.4 \\
\hline Manual anastomosis, n (\%) & $29(11)$ & $30(10)$ & 0.8 & $34(13)$ & $25(8)$ & 0.06 \\
\hline Laparoscopic, n (\%) & $119(45)$ & $99(35)$ & 0.009 & $92(36)$ & $126(43)$ & 0.1 \\
\hline $\begin{array}{l}\text { Blood loss }(\mathrm{mL}) \\
\text { mean }+\mathrm{SD}\end{array}$ & $389.1 .9+402.6$ & $361.2+340.6$ & 0.4 & $347.4+359.2$ & $397.8+380.7$ & 0.1 \\
\hline Conversion, $\mathrm{n}(\%)$ & $38(34)$ & $33(36)$ & 0.8 & $24(28)$ & $47(41)$ & 0.05 \\
\hline Loop ileostomy, n (\%) & $81(31)$ & $76(26)$ & 0.2 & $75(29)$ & $82(28)$ & 0.6 \\
\hline \multicolumn{7}{|l|}{ Post-operative outcomes } \\
\hline$A L, n(\%)$ & $24(9)$ & $29(10)$ & 0.7 & $20(8)$ & $33(11)$ & 0.2 \\
\hline $\begin{array}{l}\text { Length of stay (days), } \\
\text { mean + SD }\end{array}$ & $14.3+23.7$ & $14.7+24.8$ & 0.8 & $13.9+23.1$ & $15.0+25.3$ & 0.6 \\
\hline 30-day readmission, n (\%) & $18(7)$ & $20(7)$ & 0.9 & $19(8)$ & $19(6)$ & 0.6 \\
\hline 30-day mortality, n (\%) & $4(1.5)$ & $19(6.6)$ & 0.003 & $10(4)$ & $13(4)$ & 0.8 \\
\hline
\end{tabular}

aDefined as an SMI at the level of $\mathrm{L} 3$ of $<52.4 \mathrm{~cm}^{2} / \mathrm{m}^{2}$ in men and $<38.5 \mathrm{~cm}^{2} / \mathrm{m}^{2}$ in women.

befined as visceral fat area $>100 \mathrm{~cm}^{2}$.

BMI: body mass index; SD: standard deviation; SMI: skeletal muscle index. AL: anastomosis leak.

We found low SMI to be an independent predictor of 30-day mortality after colorectal surgery, as it has been previously described in patients with colorectal cancer ${ }^{10-13}$. Low SMI may imply a prolonged state of catabolism, impaired host immune function, and inadequate response to an inflammatory state ${ }^{14}$.

An association could not be found between $A L$ and VFA or SMI, as described in other retrospective studies $^{10,15-17}$. Even a prospective cohort study of 80 patients who underwent elective surgery for colon cancer found no relationship between SMI and any post-operative morbidity ${ }^{18}$.
In opposition to this, other authors ${ }^{10,19-23}$ have found low SMI to be a predictor of overall complications and length of stay. Herrod et al. ${ }^{21}$ evaluated psoas density as a surrogate for SMI and found that patients with smaller psoas muscles had an OR of 14.37 for $\mathrm{AL}$ and 6.33 for overall complications, though mortality was not affected. The morphologic change of the psoas has also been described as an independent risk factor for overall complications and $\mathrm{AL}$ in surgical colorectal cancer patients ${ }^{24}$. In addition, sarcopenic obesity in patients with gastrointestinal solid tumors has also been associated with ${ }^{8}$, longer length of stay, and higher readmission rates $^{24}$. In our population, 
Table 4. Variables associated with 30-day mortality on univariable and multivariable analysis

\begin{tabular}{|c|c|c|c|c|c|c|}
\hline \multirow[t]{2}{*}{ Variable } & \multicolumn{2}{|c|}{ Univariable } & \multicolumn{2}{|c|}{ Multivariable $^{\mathrm{a}}$} & \multicolumn{2}{|c|}{ Multivariable $^{b}$} \\
\hline & $\begin{array}{c}\text { OR } \\
(95 \% \mathrm{Cl})\end{array}$ & $\mathrm{p}$-value & $\begin{array}{c}\text { OR } \\
(95 \% \mathrm{Cl})\end{array}$ & $\mathrm{p}$-value & $\begin{array}{c}\text { OR } \\
(95 \% \mathrm{Cl})\end{array}$ & p-value \\
\hline Low SMlc & $\begin{array}{c}4.59 \\
(1.54-13.67)\end{array}$ & 0.006 & $\begin{array}{c}4.74 \\
(1.22-18.36)\end{array}$ & 0.02 & - & - \\
\hline L3-SMI, $\mathrm{cm}^{2} / \mathrm{m}^{2}$ & $\begin{array}{c}0.92 \\
(0.87-0.97)\end{array}$ & 0.003 & - & - & $\begin{array}{c}0.93 \\
(0.87-0.99)\end{array}$ & 0.03 \\
\hline Visceral obesity ${ }^{d}$ & $\begin{array}{c}1.12 \\
(0.48-2.60)\end{array}$ & 0.8 & - & - & - & - \\
\hline VFA, $\mathrm{cm}^{2}$ & $\begin{array}{c}1.00 \\
(0.99-1.00)\end{array}$ & 0.3 & - & - & - & - \\
\hline $\begin{array}{l}\text { Low L3-SMI and visceral } \\
\text { obesity }\end{array}$ & $\begin{array}{c}2.05 \\
(0.88-4.79)\end{array}$ & 0.09 & - & - & - & - \\
\hline $\mathrm{AL}$ & $\begin{array}{c}8.62 \\
(3.57-20.82)\end{array}$ & $<0.001$ & $\begin{array}{c}5.08 \\
(1.53-16.85)\end{array}$ & 0.008 & $\begin{array}{c}5.66 \\
(1.70-18.83)\end{array}$ & 0.005 \\
\hline Albumin, $g / d L$ & $\begin{array}{c}0.34 \\
(0.20-0.56)\end{array}$ & 0.001 & $\begin{array}{c}0.33 \\
(0.16-0.68)\end{array}$ & 0.002 & $\begin{array}{c}0.36 \\
(0.17-0.73)\end{array}$ & 0.005 \\
\hline Blood loss, $\mathrm{mL}$ & $\begin{array}{c}1.00 \\
(1.00-1.001)\end{array}$ & 0.02 & $\begin{array}{c}1.00 \\
(0.99-1.00)\end{array}$ & 0.3 & $\begin{array}{c}1.00 \\
(0.99-1.00)\end{array}$ & 0.5 \\
\hline Male sex & $\begin{array}{c}1.20 \\
(0.52-2.76)\end{array}$ & 0.7 & - & - & - & - \\
\hline Age, years & $\begin{array}{c}1.06 \\
(1.03-1.09)\end{array}$ & $<0.001$ & $\begin{array}{c}1.06 \\
(1.01-1.09)\end{array}$ & 0.007 & $\begin{array}{c}1.06 \\
(1.02-1.10)\end{array}$ & 0.003 \\
\hline Steroid use & $\begin{array}{c}5.33 \\
(1.83-15.48)\end{array}$ & 0.002 & $\begin{array}{c}4.87 \\
(1.09-21.64)\end{array}$ & 0.04 & $\begin{array}{c}4.61 \\
(1.09-19.51)\end{array}$ & 0.04 \\
\hline $\mathrm{CCl}>2$ & $\begin{array}{c}10.30 \\
(3.75-28.26)\end{array}$ & $<0.001$ & $\begin{array}{c}4.64 \\
(1.37-15.66)\end{array}$ & 0.01 & $\begin{array}{c}4.32 \\
(1.30-14.33)\end{array}$ & 0.02 \\
\hline $\mathrm{BMI}, \mathrm{kg} / \mathrm{m}^{2}$ & $\begin{array}{c}0.98 \\
(0.90-1.08)\end{array}$ & 0.8 & & & & \\
\hline Presence $>1$ anastomosis & $\begin{array}{c}1.62 \\
(0.36-7.28)\end{array}$ & 0.5 & & & & \\
\hline Loop ileostomy & $\begin{array}{c}1.09 \\
(0.44-2.71)\end{array}$ & 0.8 & & & & \\
\hline \multicolumn{7}{|l|}{ Type of surgery } \\
\hline Right hemicolectomy & Reference & 0.9 & Reference & 0.6 & Reference & 0.7 \\
\hline Left hemicolectomy & $\begin{array}{c}0.94 \\
(0.11-8.28)\end{array}$ & & $\begin{array}{c}1.65 \\
(0.14-19.49)\end{array}$ & & $\begin{array}{c}1.53 \\
(0.11-19.71)\end{array}$ & \\
\hline $\begin{array}{l}\text { Surgeries with rectal } \\
\text { anastomosis }^{\mathrm{e}}\end{array}$ & $\begin{array}{c}0.97 \\
(0.27-3.41)\end{array}$ & 0.9 & $\begin{array}{c}1.71 \\
(0.38-7.74)\end{array}$ & 0.4 & $\begin{array}{c}2.21 \\
(0.49-9.80)\end{array}$ & 0.3 \\
\hline Others & $\begin{array}{c}2.78 \\
(0.95-8.07)\end{array}$ & 0.06 & $\begin{array}{c}8.89 \\
(2.04-38.78)\end{array}$ & 0.004 & $\begin{array}{c}9.59 \\
(2.20-41.73)\end{array}$ & 0.003 \\
\hline
\end{tabular}

aConsidering low SMI,

bconsidering L3-SMI.

'Defined as a SMI at the level of $\mathrm{L} 3$ of $<52.4 \mathrm{~cm}^{2} / \mathrm{m}^{2}$ in men and $<38.5 \mathrm{~cm}^{2} / \mathrm{m}^{2}$ in women.

dDefined as VFA $>100 \mathrm{~cm}^{2}$.

eTotal colectomy, proctocolectomy, sigmoidectomy, and low anterior resection.

BMI: body mass index; $\mathrm{Cl}$ : confidence interval; OR: odds ratio; SMI: skeletal muscle index. VFA: visceral fat area; AL: anastomosis leak;

$\mathrm{CCl}$ : Charlson comorbidity index. 
Table 5. Variables associated with AL on univariable and multivariable analysis

\begin{tabular}{|c|c|c|c|c|}
\hline \multirow[t]{2}{*}{ Variable } & \multicolumn{2}{|c|}{ Univariable } & \multicolumn{2}{|c|}{ Multivariable } \\
\hline & $\begin{array}{c}\text { OR } \\
(95 \% \mathrm{Cl})\end{array}$ & p-value & $\begin{array}{c}\text { OR } \\
(95 \% \mathrm{Cl})\end{array}$ & $\mathrm{p}$-value \\
\hline Low SMla & $\begin{array}{c}1.11 \\
(0.63-1.97)\end{array}$ & 0.7 & & \\
\hline L3-SMI, $\mathrm{cm}^{2} / \mathrm{m}^{2}$ & $\begin{array}{c}1.00 \\
(0.97-1.03)\end{array}$ & 0.7 & & \\
\hline Visceral obesity ${ }^{b}$ & $\begin{array}{c}1.47 \\
(0.82-2.62)\end{array}$ & 0.2 & & \\
\hline VFA, $\mathrm{cm}^{2}$ & $\begin{array}{c}1.00 \\
(0.99-1.00)\end{array}$ & 0.1 & & \\
\hline Low L3-SMI and visceral obesity & $\begin{array}{c}1.37 \\
(0.75-2.50)\end{array}$ & 0.3 & & \\
\hline Albumin, $g / d L$ & $\begin{array}{c}0.76 \\
(0.53-1.10)\end{array}$ & 0.1 & & \\
\hline Blood loss, $\mathrm{mL}$ & $\begin{array}{c}1.00 \\
(1.00-1.001)\end{array}$ & 0.006 & $\begin{array}{c}1.00 \\
(1.00-1.001)\end{array}$ & 0.007 \\
\hline Male sex & $\begin{array}{c}1.36 \\
(0.77-2.40)\end{array}$ & 0.3 & & \\
\hline Age, years & $\begin{array}{c}1.01 \\
(0.99-1.02)\end{array}$ & 0.2 & & \\
\hline Steroid use & $\begin{array}{c}1.41 \\
(0.47-4.21)\end{array}$ & 0.5 & & \\
\hline $\mathrm{CCl}>2$ & $\begin{array}{c}1.89 \\
(1.05-3.40)\end{array}$ & 0.03 & $\begin{array}{c}2.07 \\
(1.13-3.81)\end{array}$ & 0.02 \\
\hline BMI, kg/m² & $\begin{array}{c}1.03 \\
(0.97-1.08)\end{array}$ & 0.2 & & \\
\hline Loop ileostomy & $\begin{array}{c}1.32 \\
(0.72-2.40)\end{array}$ & 0.4 & & \\
\hline \multicolumn{5}{|l|}{ Type of surgery } \\
\hline Right hemicolectomy & Reference & & Reference & \\
\hline Left hemicolectomy & $\begin{array}{c}2.86 \\
(0.90-9.10)\end{array}$ & 0.07 & $\begin{array}{c}3.36 \\
(1.03-10.89)\end{array}$ & 0.04 \\
\hline Surgeries with rectal anastomosis ${ }^{c}$ & $\begin{array}{c}1.79 \\
(0.77-4.18)\end{array}$ & 0.2 & $\begin{array}{c}1.96 \\
(0.83-4.65)\end{array}$ & 0.1 \\
\hline Others & $\begin{array}{c}3.12 \\
(1.39-6.97)\end{array}$ & 0.005 & $\begin{array}{c}3.26 \\
(1.44-7.37)\end{array}$ & 0.004 \\
\hline
\end{tabular}

aDefined as a SMI at the level of $\mathrm{L} 3$ of $<52.4 \mathrm{~cm}^{2} / \mathrm{m}^{2}$ in men and $<38.5 \mathrm{~cm}^{2} / \mathrm{m}^{2}$ in women.

bDefined as VFA $>100 \mathrm{~cm}^{2}$. cTotal colectomy, proctocolectomy, sigmoidectomy, and low anterior resection.

BMI: body mass index; $\mathrm{Cl}$ : confidence interval; OR: odds ratio; SMI: skeletal muscle index. VFA: visceral fat area; AL: anastomosis leak;

$\mathrm{CCl}$ : Charlson comorbidity index.

visceral obesity in conjunction with low SMI was not related to any adverse outcomes.

Other predictive factors for 30-day mortality besides skeletal muscle in multivariate analysis were AL, preoperative albumin levels, age, $\mathrm{CCl}>2$, "other" types of surgery, and steroid use. AL is a very threatening complication in colorectal surgery, whose association with 30-day has been previously described ${ }^{25}$.

Concerning pre-operative albumin, patients with lower albumin may have a poorer nutritional status that 
relates to negative outcomes ${ }^{26}$. Patients with greater estimated blood loss may have had more difficult operative courses, linked to longer operative times and more blood transfusions. All these elements may be related to adverse post-operative outcomes and, thus, more mortality. Moreover, blood loss has been related to a depleting effect in the immune system ${ }^{27,28}$. Steroid use may be associated with mortality because of impaired tissue healing and immune response ${ }^{29}$. Mortality is also expected to be more prevalent among older people, with more comorbidities as our study suggested. The increased 30-day mortality among patients who had other types of surgery (including stoma closure and segmental colonic resection) cannot be explained, as it is expected that surgeries with rectal anastomosis are more challenging ${ }^{25}$. However, since these surgeries were also related to AL, a high 30-day mortality is expected.

Visceral obesity has been related to adverse outcomes in colorectal surgery ${ }^{30-33}$, including a higher incidence of systemic complications presumably caused by longer operative times, a higher frequency of metabolic, cardiovascular comorbidities ${ }^{34}$, and more operative blood loss ${ }^{35}$. At least two meta-analyses by Yang et al. ${ }^{36}$ and Cakir et al. ${ }^{4}$ found that post-operative complications in laparoscopic procedures were more frequent in patients with visceral obesity. However, we could not corroborate these data. Visceral obesity was not associated with mortality or AL. Other authors have also found no increased morbidity among patients with visceral obesity ${ }^{37-39}$, and the reason behind this discrepancy may be that visceral obesity mainly seems to affect laparoscopic approaches, and only $40 \%$ of the cases in our cohort were laparoscopic. A meta-analysis on the association between visceral obesity and AL could not draw appropriate conclusions due to the heterogeneity between studies, but a tendency against an association between $\mathrm{AL}$ and visceral obesity was established ${ }^{40}$.

Our study has several limitations, the most important one being its retrospective nature. Although an association between low SMI and mortality was found, prospective studies are needed to confirm the association and explore whether preconditioning and muscle mass improvement may reduce mortality. Another limitation is that our study only evaluates
SMI. Muscle function was not considered in this analysis, so its interaction with surgical outcomes could not be described. Finally, the number of outcomes in our study was limited, and therefore, the results of the multivariable analysis should be interpreted with caution.

In conclusion, SMI, but not visceral obesity, was independently associated with 30-day mortality. Neither low SMI nor visceral obesity was associated with AL. The role of preconditioning in elective surgeries to improve outcomes needs to be further explored.

\section{Supplementary Data}

Supplementary data are available at Revista de Investigación Clínica online (www.clinicalandtranslationalinvestigation.com). These data are provided by the corresponding author and published online for the benefit of the reader. The contents of supplementary data are the sole responsibility of the authors.

\section{REFERENCES}

1. Maurovich-Horvat P, Massaro J, Fox CS, Moselewski F, O'Donnell C], Hoffmann U. Comparison of anthropometric, area-and volume-based assessment of abdominal subcutaneous and visceral adipose tissue volumes using multi-detector computed tomography. Int J Obes. 2006;31:500-6.

2. Perthen JE, Ali T, McCulloch D, Navidi M, Phillips AW, Sinclair RC, et al. Intra-and interobserver variability in skeletal muscle measurements using computed tomography images. Eur J Radiol. 2018;109:142-6.

3. Yamamoto N, Fujii S, Sato T, Oshima T, Rino Y, Kunisaki C, et al. Impact of body mass index and visceral adiposity on outcomes in colorectal cancer. Asia Pac J Clin Oncol. 2012;8:337-45.

4. Cakir H, Heus C, van der Ploeg TJ, Houdijk AP. Visceral obesity determined by CT scan and outcomes after colorectal surgery; a systematic review and meta-analysis. Int J Colorectal Dis. 2015;30:875-82.

5. Lieffers JR, Bathe OF, Fassbender $K$, Winget $M$, Baracos VE. Sarcopenia is associated with postoperative infection and delayed recovery from colorectal cancer resection surgery. $\mathrm{Br}$ ] Cancer. 2012;107:931-6.

6. Peterson SJ, Braunschweig CA. Prevalence of sarcopenia and associated outcomes in the clinical setting. Nutr Clin Pract. 2016;31:40-8

7. Schneider CA, Rasband WS, Eliceiri KW. NIH image to Image]: 25 years of image analysis. Nat Methods. 2012;9:671-5.

8. Prado CM, Lieffers JR, McCargar LJ, Reiman T, Sawyer MB, Martin $L$, et al. Prevalence and clinical implications of sarcopenic obesity in patients with solid tumours of the respiratory and gastrointestinal tracts: a population-based study. Lancet Oncol. 2008;9:629-35.

9. Viera AJ, Garrett JM. Understanding interobserver agreement: the kappa statistic. Fam Med. 2005;37:360-3

10. Reisinger KW, Van Vugt JL, Tegels JJ, Snijders C, Hulsewé KW Hoofwijk AG, et al. Functional compromise reflected by sarcopenia, frailty, and nutritional depletion predicts adverse postoperative outcome after colorectal cancer surgery. Ann Surg. 2015;261:345-52. 
11. Dolan DR, Knight KA, Maguire S, Moug SJ. The relationship between sarcopenia and survival at 1 year in patients having elective colorectal cancer surgery. Tech Coloproctol. 2019; 23:877-85.

12. Sun G, Li Y, Peng Y, Lu D, Zhang F, Cui X, et al. Can sarcopenia be a predictor of prognosis for patients with non-metastatic colorectal cancer? A systematic review and meta-analysis. Int J Colorectal Dis. 2018;33:1419-27.

13. van Vugt JL, van den Braak RR, Lalmahomed ZS, Vrijland WW, Dekker JW, Zimmerman DD, et al. Impact of low skeletal muscle mass and density on short and long-term outcome after resection of stage I-III colorectal cancer. Eur J Surg Oncol. 2018; 44:1354-60.

14. Malietzis G, Johns N, Al-Hassi $\mathrm{HO}$, Knight SC, Kennedy RH Fearon KC, et al. Low muscularity and myosteatosis is related to the host systemic inflammatory response in patients undergoing surgery for colorectal cancer. Ann Surg. 2016;263:320-5.

15. Nakanishi R, Oki E, Sasaki S, Hirose K, Jogo T, Edahiro K, et al. Sarcopenia is an independent predictor of complications after colorectal cancer surgery. Surg Today. 2018;48:151-7.

16. Kobayashi A, Kaido T, Hamaguchi Y, Okumura S, Shirai H, Kamo $\mathrm{N}$, et al. Impact of visceral adiposity as well as sarcopenic factors on outcomes in patients undergoing liver resection for colorectal liver metastases. World J Surg. 2018:42:1180-91.

17. Chen WZ, Chen XD, Ma LL, Zhang FM, Lin J, Zhuang C Le, et al. Impact of visceral obesity and sarcopenia on short-term outcomes after colorectal cancer surgery. Dig Dis Sci. 2018; 63:1620-30.

18. van der Kroft G, Bours DM, Janssen-Heijnen DM, van Berlo DC, Konsten DJ. Value of sarcopenia assessed by computed tomography for the prediction of postoperative morbidity following oncological colorectal resection: a comparison with the malnutrition screening tool. Clin Nutr ESPEN. 2018;24:114-9.

19. Peng PD, van Vledder MG, Tsai $S$, de Jong MC, Makary $M, N g$ J, et al. Sarcopenia negatively impacts short-term outcomes in patients undergoing hepatic resection for colorectal liver metastasis. HPB. 2011;13:439-46.

20. Robinson TN, Wu DS, Stiegmann GV, Moss M. Frailty predicts increased hospital and six-month healthcare cost following colorectal surgery in older adults. Am J Surg. 2011;202:511-4.

21. Herrod PJ, Boyd-Carson H, Doleman B, Trotter J, Schlichtemeier S, Sathanapally G, et al. Quick and simple; psoas density measurement is an independent predictor of anastomotic leak and other complications after colorectal resection. Tech Coloproctol. 2019;23:129-34

22. Hanaoka M, Yasuno M, Ishiguro M, Yamauchi S, Kikuchi A, Tokura $\mathrm{M}$, et al. Morphologic change of the psoas muscle as a surrogate marker of sarcopenia and predictor of complications after colorectal cancer surgery. Int J Colorectal Dis. 2017; 32:847-56

23. Tan KY, Kawamura YJ, Tokomitsu A, Tang T. Assessment for frailty is useful for predicting morbidity in elderly patients undergoing colorectal cancer resection whose comorbidities are already optimized. Am J Surg. 2012;204:139-43.

24. Martin L, Hopkins J, Malietzis G, Jenkins JT, Sawyer MB, Brisebois $\mathrm{R}$, et al. Assessment of computed tomography (CT)-defined muscle and adipose tissue features in relation to shortterm outcomes after elective surgery for colorectal cancer: a multicenter approach. Ann Surg Oncol. 2018;25:2669-80.
25. Midura EF, Hanseman D, Davis BR, Atkinson SJ, Abbott DE, Shah $\mathrm{SA}$, et al. Risk factors and consequences of anastomotic leak after colectomy: a national analysis. Dis Colon Rectum. 2015 58:333-8.

26. Chiang JM, Chang CJ, Jiang SF, Yeh CY, You JF, Hsieh PS, et al. Pre-operative serum albumin level substantially predicts postoperative morbidity and mortality among patients with colorectal cancer who undergo elective colectomy. Eur J Cancer Care. 2017;26:12403

27. Angele MK, Faist E. Clinical review: immunodepression in the surgical patient and increased susceptibility to infection. Crit Care. 2002;6:298-305.

28. Yago $H$, Yoshii $H$, Naiki M, Suehiro S. Stress and murine NK cell function: the role of blood loss. J Clin Lab Immunol. 1992; $37: 123-32$

29. Moghadamyeghaneh Z, Hanna MH, Blondet JJ, Carmichael JC, Mills SD, Pigazzi A, et al. Impact of chronic steroid use on outcomes of colorectal surgery. Am J Surg 2015;210:1003-9.

30. Kang J, Baek SE, Kim T, Hur H, Min BS, Lim JS, et al. Impact of fat obesity on laparoscopic total mesorectal excision: more reliable indicator than body mass index. Int J Colorectal Dis. 2012;27:497-505

31. Tappouni R, Mathew P, Connelly TM, Luke F, Messaris E. Measurement of visceral fat on preoperative computed tomography predicts complications after sigmoid colectomy for diverticular disease. Am J Surg. 2015;210:285-90

32. Ozoya OO, Siegel EM, Srikumar T, Bloomer AM, DeRenzis A, Shibata D. Quantitative assessment of visceral obesity and postoperative colon cancer outcomes. J Gastrointest Surg. 2017;21:534-42

33. Park BK, Park JW, Ryoo SB, Jeong SY, Park KJ, Park JG. Effect of visceral obesity on surgical outcomes of patients undergoing laparoscopic colorectal surgery. World J Surg. 2015;39:2343-53.

34. Ishii $Y$, Hasegawa $H$, Nishibori $H$, Watanabe M, Kitajima M. Impact of visceral obesity on surgical outcome after laparoscopic surgery for rectal cancer. Br J Surg. 2005;92:1261-2.

35. Seki Y, Ohue M, Sekimoto M, Takiguchi S, Takemasa I, Ikeda M, et al. Evaluation of the technical difficulty performing laparoscopic resection of a rectosigmoid carcinoma: visceral fat reflects technical difficulty more accurately than body mass index. Surg Endosc Other Interv Tech. 2007;21:929-34.

36. Yang $T$, Wei M, He Y, Deng X, Wang Z. Impact of visceral obesity on outcomes of laparoscopic colorectal surgery: a metaanalysis. ANZ J Surg. 2015;85:507-13

37. Moon HG, Ju YT, Jeong CY, Jung EJ, Lee YJ, Hong SC, et al. Visceral obesity may affect oncologic outcome in patients with colorectal cancer. Ann Surg Oncol. 2008;15:1918-22.

38. Cecchini S, Cavazzini E, Marchesi F, Sarli L, Roncoroni L. Computed tomography volumetric fat parameters versus body mass index for predicting short-term outcomes of colon surgery. World J Surg. 2011;35:415-23

39. Ballian N, Lubner MG, Munoz A, Harms BA. Heise CP, Foley EF, et al. Visceral obesity is associated with outcomes of total mesorectal excision for rectal adenocarcinoma. J Surg Oncol. 2012;105:365-70

40. Malietzis G, Aziz O, Bagnall NM, Johns N, Fearon KC, Jenkins JT. The role of body composition evaluation by computerized tomography in determining colorectal cancer treatment outcomes: a systematic review. Eur J Surg Oncol. 2015;41:186-96. 